\title{
Gestión administrativa y desempeño laboral en una entidad educativa de Arequipa en la coyuntura de COVID-19
}

\author{
Administrative management and labor performance in an educational entity \\ in Arequipa in the situation of COVID-19
}

\author{
Diego Gonzalo Fernandez Sanchez \\ Institución Educativa Andenes de Chilina \\ Arequipa, Perú \\ dfernandezsa@unsa.edu.pe \\ https://orcid.org/0000-0003-1820-5023
}

\section{RESUMEN}

El propósito de la presente investigación fue definir la relación existente entre las variables de gestión administrativa y el desempeño laboral en docentes de la Institución Educativa “Andenes de Chilina”, Arequipa, 2020. Metodológicamente, es una investigación de tipo pura, también conocida como fundamental. El nivel de investigación fue correlacional, donde se determinó la relación entre las dos variables objeto de estudio. Se trabajó bajo el método hipotético-deductivo con enfoque cuantitativo y de corte transversal, debido a que se realizó la medición una sola vez en el tiempo. Se contó con una población muestral de 28 docentes de ambos sexos, los cuales se encuentran dentro de los niveles educativos básicos (inicial, primaria y secundaria). En los resultados obtenidos, según la prueba estadística de correlación de Pearson aplicado a nuestras variables en estudio: gestión administrativa y desempeño laboral, se consiguió un $r=0.834$ (correlación positiva alta) con un nivel 0.000 de significancia, el cual fue menor que el valor esperado ( $\mathrm{p}<0.05)$. Fue por esta razón que se aceptó la hipótesis alterna, rechazando la hipótesis nula formulada. Se concluyó que existe una relación positiva y significativa entre gestión administrativa y desempeño laboral docente en la Institución Educativa “Andenes de Chilina”, Arequipa, 2020.

Palabras clave: Desempeño laboral, docente, gestión administrativa, institución educativa.

\begin{abstract}
The objective of this investigation was to define the relationship between administrative management variables and work performance in teachers of the "Andenes de Chilina" Educational Institution, Arequipa, 2020. Methodologically, it is a pure research, also known as fundamental. The research level was correlational, in which the relationship between the two variables under study was determined. We worked under the hypothetical-deductive method with a quantitative and crosssectional approach, because the measurement was carried out only once in time. The sample population consisted of 28 teachers of both sexes, who are in the basic educational levels (initial, primary and secondary). The results obtained, according to Pearson's correlation statistical test applied to our variables under study: administrative management and work performance, showed an $r=0.834$ (high positive correlation) with a significance level of 0.000 , which was lower than the expected value $(\mathrm{p}<0.05)$. For this reason, the alternative hypothesis was accepted, rejecting the null hypothesis. The conclusion was that there is a positive and significant relationship between administrative management and teacher job performance at the "Andenes de Chilina" Educational Institution, Arequipa, 2020.
\end{abstract}

Keywords: Job performance, teacher, administrative management, educational institution.

Este es un artículo de acceso abierto, distribuido bajo los términos de la Licencia Creative Commons Atribución 4.0 Internacional (CC BY 4.0). https://creativecommons.org/licenses/by/4.0/deed.es 


\section{INTRODUCCIÓN}

La imagen que se tiene de la globalización está sumergida en diferentes escenarios sociales, obligándonos a adquirir rápidas respuestas a estos acontecimientos. Por esta razón, es importante que la educación avance junto con esta transformación, no quedándose de lado como nuestro sistema educativo actual que tiene diferentes deficiencias que debe hacer frente para el bienestar de la población (Chávez Albarrán \& Olivos Sánchez, 2019).

Benites y Castillo (2018) mencionan que la gestión administrativa en las instituciones educativas es uno de los factores principales para que estas tengan éxito, no obstante, en muchos casos lo que impide la consecución de las buenas metas del sistema educativo lo conforma el tipo de gestión administrativa que se ejecutaría, pudiendo catalogarse esta de buena, regular o mala. Es importante también reconocer que la administración es una de las prioridades en el proceso educativo, por considerarse primordial para su correcto desempeño, la administración otorgará la excelencia que se requiere por la estructura que brinda, la organización, filosofía, y por la calidad que el docente ejecuta en las aulas. La gestión administrativa tiene como base: la organización, la delegación de funciones y la búsqueda de rentabilidad, metas que debe lograrse a través del desempeño directivo, que es el eje base de todas las organizaciones. Asimismo, reconoce que para el buen desarrollo de las diversas empresas, el desempeño y la administración son variables de suma importancia, este es el fin que buscará estudiar la siguiente investigación.

Según Carbajal (2019), existen determinadas situaciones que condicionan el desempeño de las tareas y la consecución de objetivos en los diversos centros educativos. Estos escenarios están determinados por diferentes interacciones que se van dando entre estas y las múltiples características personales de los miembros y las características grupales que puedan diferenciarse. El logro de los objetivos va a depender en gran medida de la eficacia que se logre en la ejecución de las tareas y de la conducta.

Otro de los factores importantes para poder definir la calidad que posee una institución educativa es el rol que cumplen los docentes junto a las diversas condiciones de la gestión educativa en la que ellos se puedan desempeñar (Meza, 2019).

Por otro lado, Vaillant (2009) menciona que actualmente en la mayoría de los países de Latinoamérica aún se tiene en cuenta la variedad de las situaciones regionales, caracterizándose por:

- Los estímulos presentes para elegir la profesión de educador son mínimas, sumándose además las deficientes condiciones de trabajo y los graves problemas en la estructura educativa (remuneración e incentivos).

- El poco esfuerzo de algunos docentes por continuar educándose y preparándose, quedando así desligados del avance a gran escala de la educación en otros países desarrollados.

- La falta de actuación como mecanismos de mejora de la gestión institucional y la evaluación de los docentes.

Por su parte, Chávez \& Olivos (2019) señalan que el desempeño docente es uno de los problemas latentes en el Perú, mostrándose esta deficiencia en la práctica pedagógica cuando los educadores tienen que enfrentar dificultades en el desarrollo de diversos procesos, incluidos los didácticos y pedagógicos. En los últimos años, el Perú viene atravesando cambios significativos en su sistema educativo. Se busca poder lograr diversos modelos de calidad, donde el docente sumado a la mejora de diversos factores del ámbito institucional contribuirá a que la educación pueda lograr el desarrollo que se espera.

En la entidad "Andenes de Chilina" de Arequipa, colegio privado y de educación básica regular, se detectaron ciertas deficiencias en la gestión administrativa, como problemas en la organización y una falta de comprensión de las funciones. Cabe mencionar que antes de la coyuntura COVID-19 se presentaron descoordinaciones en lo que respecta a eventos externos.

Es por ello que el problema principal de la investigación fue: ¿Cuál es la relación entre la gestión administrativa y desempeño laboral de los docentes en la institución educativa "Andenes de Chilina", Arequipa, 2020? Los problemas específicos son: ¿Existe relación entre planificación de la gestión administrativa y el desempeño laboral en los docentes de una entidad educativa de Arequipa en la coyuntura de COVID-19? ¿Existe relación entre organización de la gestión administrativa y el desempeño laboral en los docentes de una entidad educativa 
de Arequipa en la coyuntura de COVID-19? ¿Existe relación entre dirección de la gestión administrativa y el desempeño laboral en los docentes de una entidad educativa de Arequipa en la coyuntura de COVID-19? ¿Existe relación entre control de la gestión administrativa y el desempeño laboral en los docentes de una entidad educativa de Arequipa en la coyuntura de COVID-19?

Por lo tanto, el presente trabajo de investigación se desarrolló en la ciudad de Arequipa, con el objetivo de aportar a la sociedad educativa y local sobre la manera en que se relaciona la gestión administrativa y el desempeño laboral docente en la Institución Educativa “Andenes de Chilina”, Arequipa, 2020.

Es así que se plantea la hipótesis general de la investigación: "Es probable que la gestión administrativa se relacione directa y significativamente con el desempeño laboral en una entidad educativa de Arequipa en la coyuntura de COVID-19". Además, se plantearon las siguientes hipótesis específicas: "Es probable que la planificación de la gestión administrativa se relacione directa y significativamente con el desempeño laboral en una entidad educativa de Arequipa en la coyuntura de COVID-19", "Es probable que la organización de la gestión administrativa se relacione directa y significativamente con el desempeño laboral en una entidad educativa de Arequipa en la coyuntura de COVID-19", "Es probable que la dirección de la gestión administrativa se relacione directa y significativamente con el desempeño laboral en una entidad educativa de Arequipa en la coyuntura de COVID-19", "Es probable que el control de la gestión administrativa se relacione directa y significativamente con el desempeño laboral en una entidad educativa de Arequipa en la coyuntura de COVID$19 "$.

\section{MATERIALY MÉTODOS}

El desarrollo de la investigación es de tipo pura o fundamental, a partir de lo mencionado por Hernández, Fernández y Baptista (2014), buscando poder obtener respuestas a una curiosidad científica, siendo en esta ocasión conocer el grado de relación que se tienen entre gestión administrativa y desempeño laboral docente en una institución educativa.

En relación a ello, la investigación se da a un nivel correlacional (Hernández, Fernándezy Baptista, 2014), la cual tiene como fin conocer el grado de relación presente entre dos o más variables de estudio en un ambiente específico, siendo en este caso las variables gestión administrativa y satisfacción laboral.

El método empleado fue el hipotético deductivo (Rodríguez \& Pérez, 2017), este tipo de método toma como base una hipótesis inferida de principios o leyes la cual es sugerida por los datos empíricos. Este tipo de método aplica reglas de la deducción, llegando a obtener predicciones que luego serán sometidas a verificación empírica, para comprobar si hay o no correspondencia con los hechos. Además, se empleó un enfoque cuantitativo que, según Hernández, Fernández \& Baptista (2014), busca analizar una realidad objetiva a partir de controles numéricos y el análisis estadístico, todo esto con el fin de obtener predicciones del problema señalado.

De la misma manera, la investigación solo va a recolectar y analizar datos de un periodo de tiempo específico, por lo que se considera un estudio de corte transversal.

La técnica empleada fue la encuesta, se utilizaron dos instrumentos mediante la aplicación de un cuestionario de manera virtual debido a la coyuntura del COVID-19. Para la gestión administrativa, se usó el Cuestionario de Gestión Administrativa de Tacuche Mariño (2018); y para desempeño laboral, se usó el Cuestionario de Desempeño Laboral de Williams y Anderson (1991). Ambos instrumentos están validados, y respecto a la confiabilidad poseen un Alfa de Cronbach de 0.745 y 0.971 respectivamente. La investigación se realizó con una muestra de 28 docentes, para el procesamiento de los datos se empleó el software SPSS, haciendo uso del programa Microsoft Excel para el análisis de la información. Como técnica estadística para el análisis de la información se trabajó con el Coeficiente de Correlación de Pearson. 
Fernandez, D.

\section{RESULTADOS}

\section{Características de la muestra}

Tabla1

Género de la muestra

\begin{tabular}{llccll} 
& Gérero & Frecuencia & Porcentaje & $\begin{array}{l}\text { Porcentaje } \\
\text { válido }\end{array}$ & $\begin{array}{l}\text { Porcentaje } \\
\text { acumulado }\end{array}$ \\
\hline \multirow{3}{*}{ Válido } & Femenino & 19 & $67.86 \%$ & $67.86 \%$ & $67.86 \%$ \\
& Masculino & 9 & $32.14 \%$ & $32.14 \%$ & $100 \%$ \\
& Total & 28 & $100 \%$ & $100 \%$ & \\
\hline
\end{tabular}

\section{Figura 1}

Género de la muestra

\begin{tabular}{|ccc|} 
& \multicolumn{2}{c|}{ Género } \\
$80.00 \%$ & $67.86 \%$ & \\
\cline { 2 - 3 } & & \\
$40.00 \%$ & & $32.14 \%$ \\
\hline $20.00 \%$ & & \\
$0.00 \%$ & & Masculino \\
\hline
\end{tabular}

\section{Tabla2}

Edad de la muestra

\begin{tabular}{llllll}
\multicolumn{1}{l}{ Laaa } & rrecuencia & rorcentaje & $\begin{array}{l}\text { Porcentaje } \\
\text { válido }\end{array}$ & $\begin{array}{l}\text { Porcentaje } \\
\text { acumulado }\end{array}$ \\
\hline \multirow{3}{*}{ Válido } & Menos de 30 años & 4 & $14.29 \%$ & $14.29 \%$ & $14.29 \%$ \\
& De 31 a 40 años & 15 & $53.57 \%$ & $53.57 \%$ & $67.86 \%$ \\
& De 41 a 50 años & 8 & $28.57 \%$ & $28.57 \%$ & $96.43 \%$ \\
& Más de 50 años & 1 & $3.57 \%$ & $3.57 \%$ & $100 \%$ \\
& Total & 28 & $100 \%$ & $100 \%$ & \\
\hline
\end{tabular}

Figura 2

Edad de la muestra

\section{Edad}

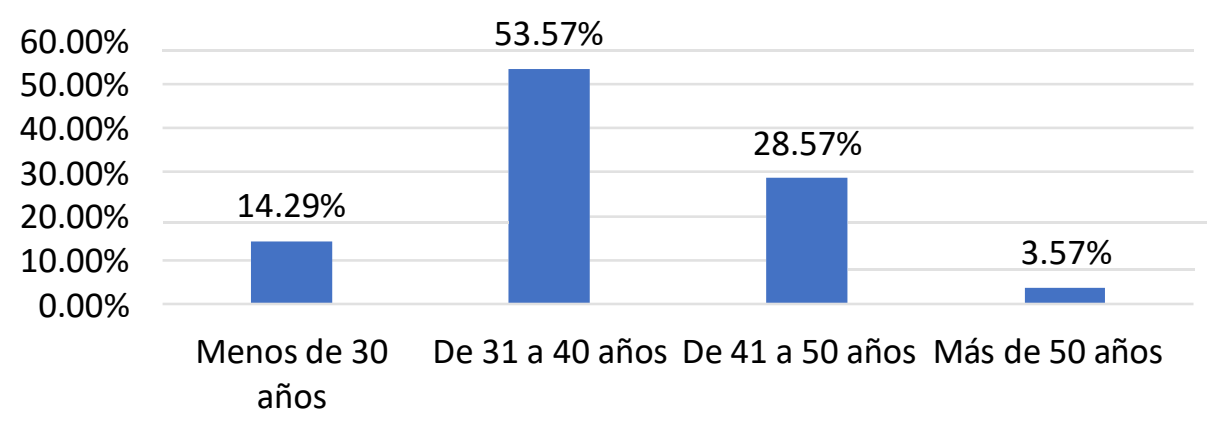


Tabla 3

Tiempo de servicio

\begin{tabular}{llccll} 
& recuencia & rorcentaje & $\begin{array}{l}\text { Porcentaje } \\
\text { válido }\end{array}$ & $\begin{array}{l}\text { Porcentaje } \\
\text { acumulado }\end{array}$ \\
\hline \multirow{3}{*}{ Válido } & Menos de 1 año & 8 & $28.57 \%$ & $28.57 \%$ & $28.57 \%$ \\
& De 1 a 3 años & 4 & $14.29 \%$ & $14.29 \%$ & $42.86 \%$ \\
& Más de 3 años & 16 & $57.14 \%$ & $57.14 \%$ & $100 \%$ \\
& Total & 28 & $100 \%$ & $100 \%$ & \\
\hline
\end{tabular}

\section{Figura 3}

Tiempo de servicio

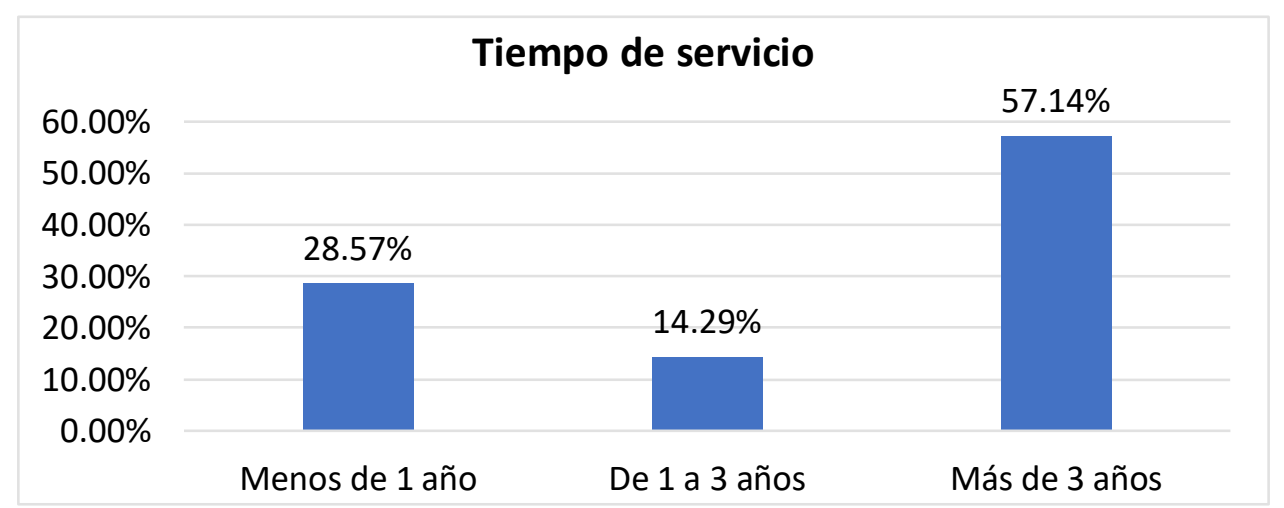

\section{Tabla 4}

Nivel en que laboran los docentes

\begin{tabular}{llllll} 
& Nivel & Frecuencia & Porcentaje & $\begin{array}{l}\text { Porcentaje } \\
\text { válido }\end{array}$ & $\begin{array}{l}\text { Porcentaje } \\
\text { acumulado }\end{array}$ \\
\hline \multirow{3}{*}{ Válido } & Inicial & 6 & $21.43 \%$ & $21.43 \%$ & $21.43 \%$ \\
& Primaria & 10 & $35.71 \%$ & $35.71 \%$ & $57.14 \%$ \\
& Secundaria & 11 & $39.29 \%$ & $39.29 \%$ & $96.43 \%$ \\
& Los tres niveles & 1 & $3.57 \%$ & $3.57 \%$ & 100 \\
& Total & 28 & 100 & 100 & \\
\hline
\end{tabular}

\section{Figura 4}

Nivel en que laboran los docentes

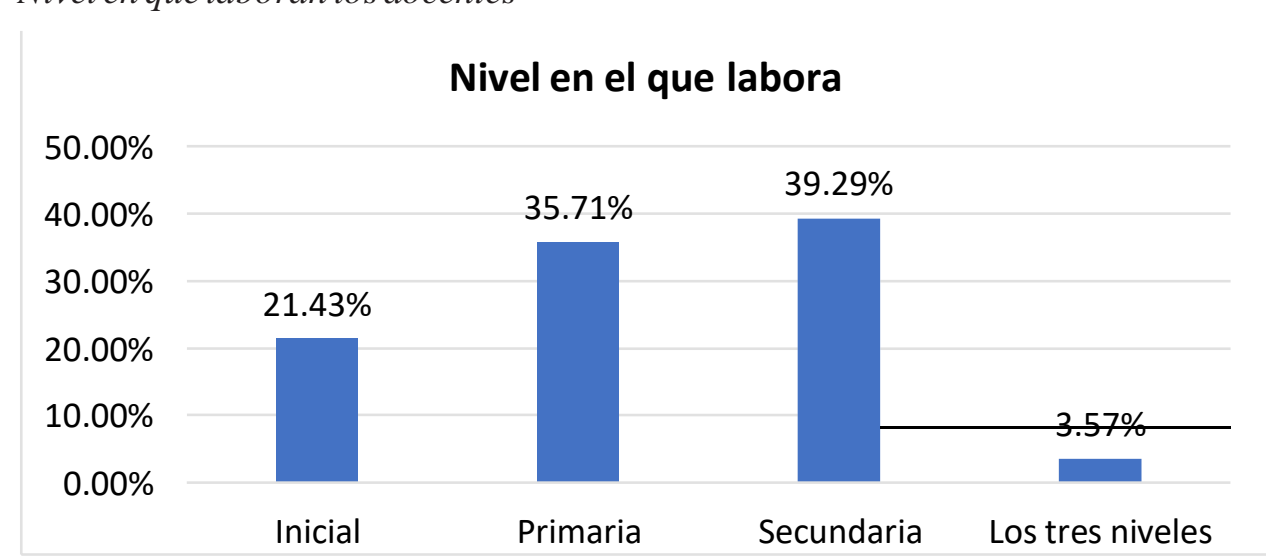




\section{Tabla 5}

Área académica

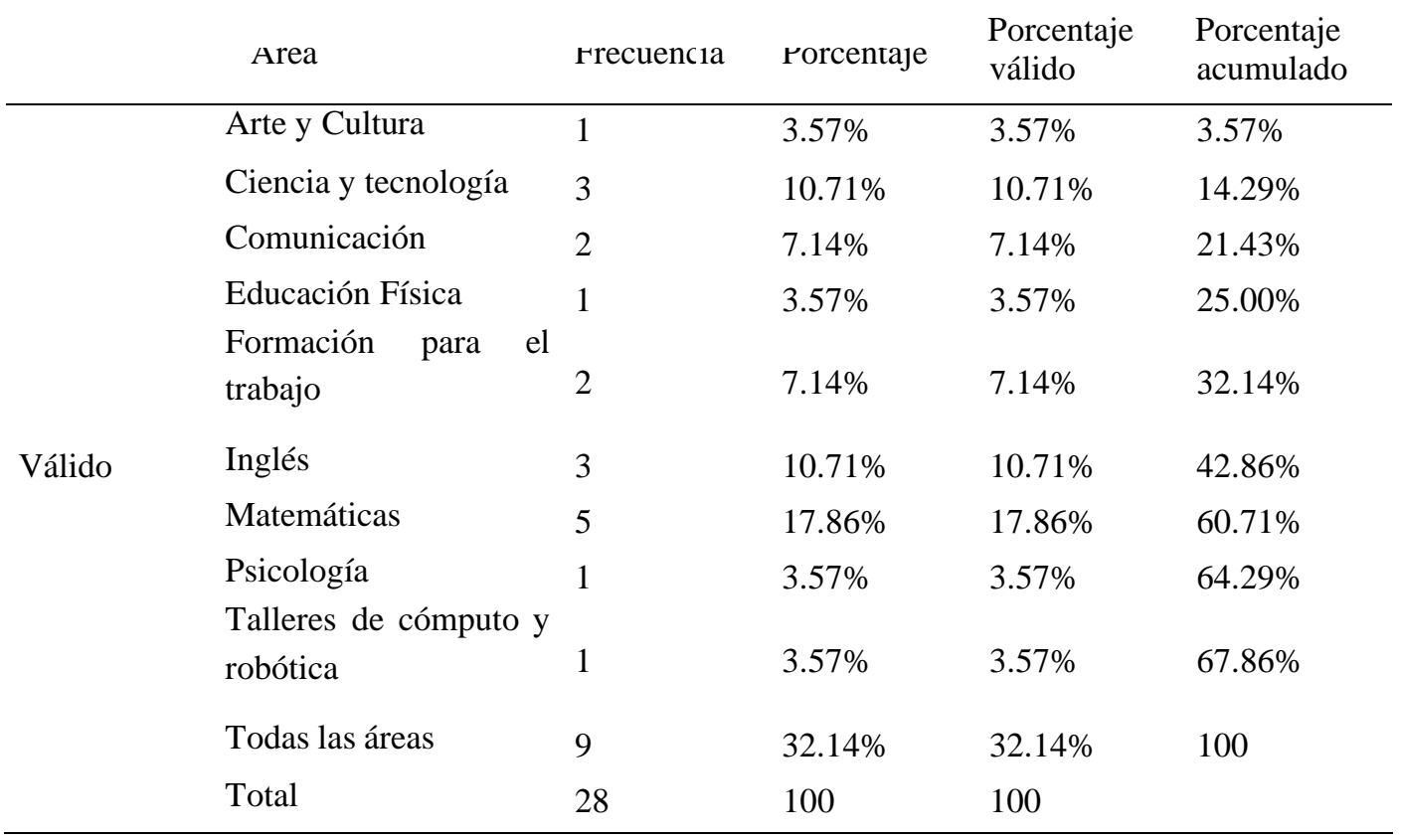

\section{Figura 5}

Área académica

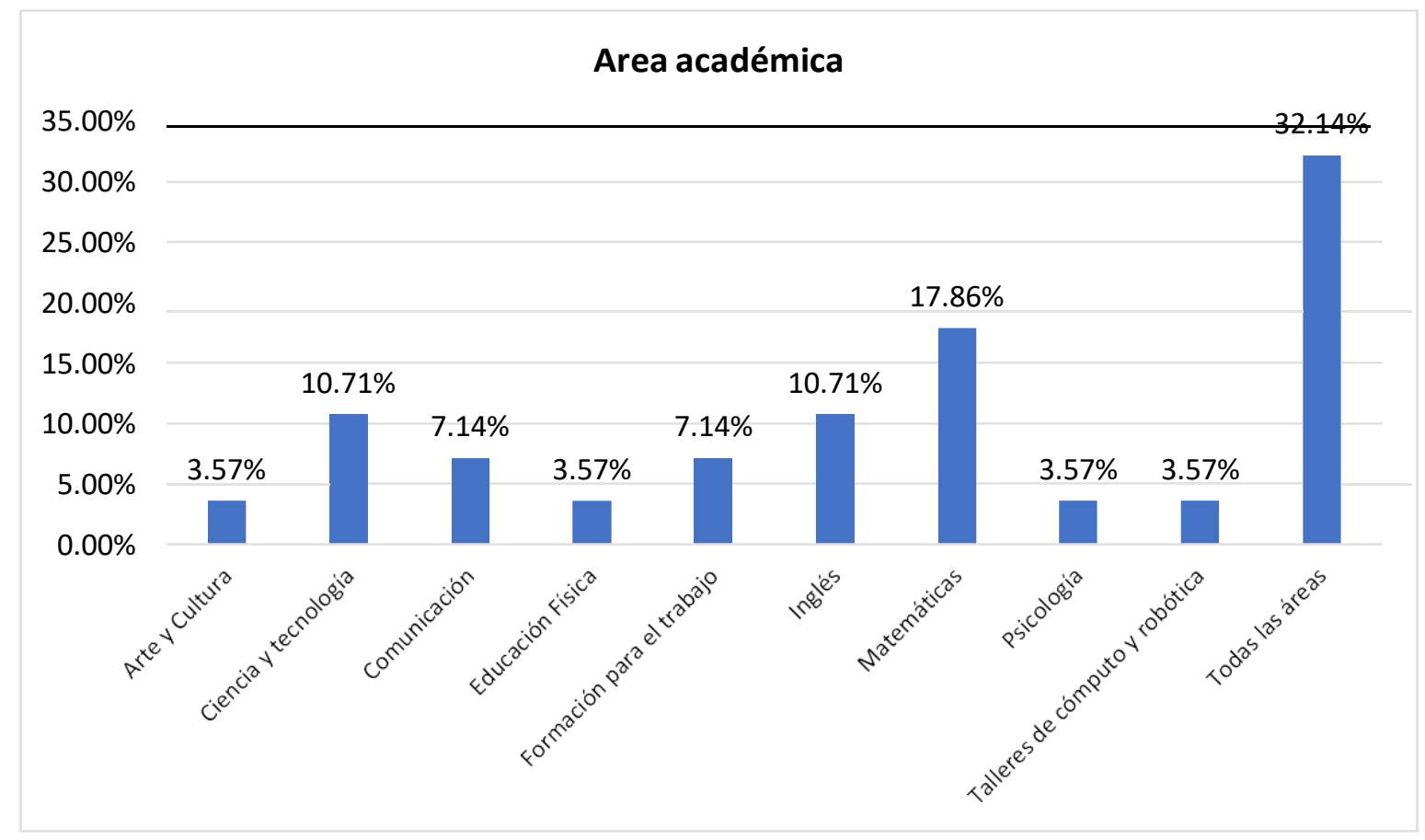




\section{Gestión Administrativa}

Tabla 6

Nivel de gestión administrativa en los docentes de una institución educativa

\begin{tabular}{llllll}
\hline Gestión Administrativa & & & & \\
\hline \multirow{6}{*}{ Válido } & rrecuencia & rorcentaje & $\begin{array}{l}\text { Porcentaje } \\
\text { válido }\end{array}$ & $\begin{array}{l}\text { Porcentaje } \\
\text { acumulado }\end{array}$ \\
& Bajo & 3 & $10.71 \%$ & $10.71 \%$ & $10.71 \%$ \\
& Medio & 5 & $17.86 \%$ & $17.86 \%$ & $28.57 \%$ \\
& Alto & 20 & $71.43 \%$ & $71.43 \%$ & $100 \%$ \\
\hline
\end{tabular}

\section{Figura 6}

Nivel de gestión administrativa en los docentes de una institución educativa

\section{Gestión Administrativa}

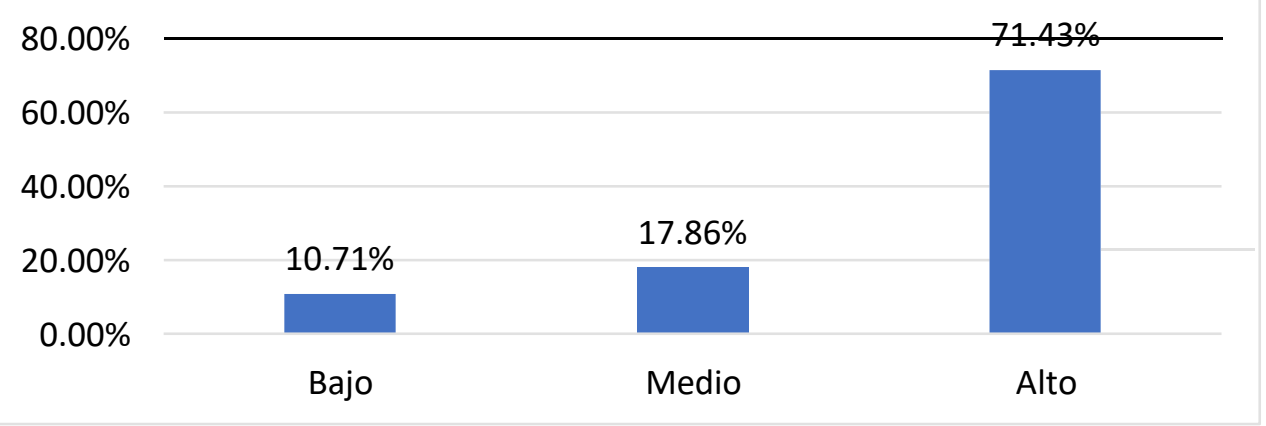

De la tabla y figura anteriores, se observó que en general el nivel de gestión administrativa es bueno, donde un $71.43 \%$ señaló un nivel alto, seguido por un $17.86 \%$ que indicó un nivel medio; y por último un $10.71 \%$ señaló nivel bajo.

\section{Tabla 7}

Nivel de la dimensión gestión de planificación en los docentes de una institución educativa

\begin{tabular}{llllll}
\hline Gestión de Planificación & & & & \\
\hline & Frecuencia & Porcentaje & $\begin{array}{l}\text { Porcentaje } \\
\text { válido }\end{array}$ & $\begin{array}{l}\text { Porcentaje } \\
\text { acumulado }\end{array}$ \\
\hline \multirow{3}{*}{ Válido } & Bajo & 3 & $10.71 \%$ & $10.71 \%$ & $10.71 \%$ \\
& Avicuiv & 5 & $17.86 \%$ & $17.86 \%$ & $28.57 \%$ \\
& Alto & 20 & $71.43 \%$ & $71.43 \%$ & $100 \%$ \\
& Total & 28 & $100 \%$ & $100 \%$ & \\
\hline
\end{tabular}




\section{Figura 7}

Nivel de la dimensión gestión de planificación en los docentes de una institución educativa

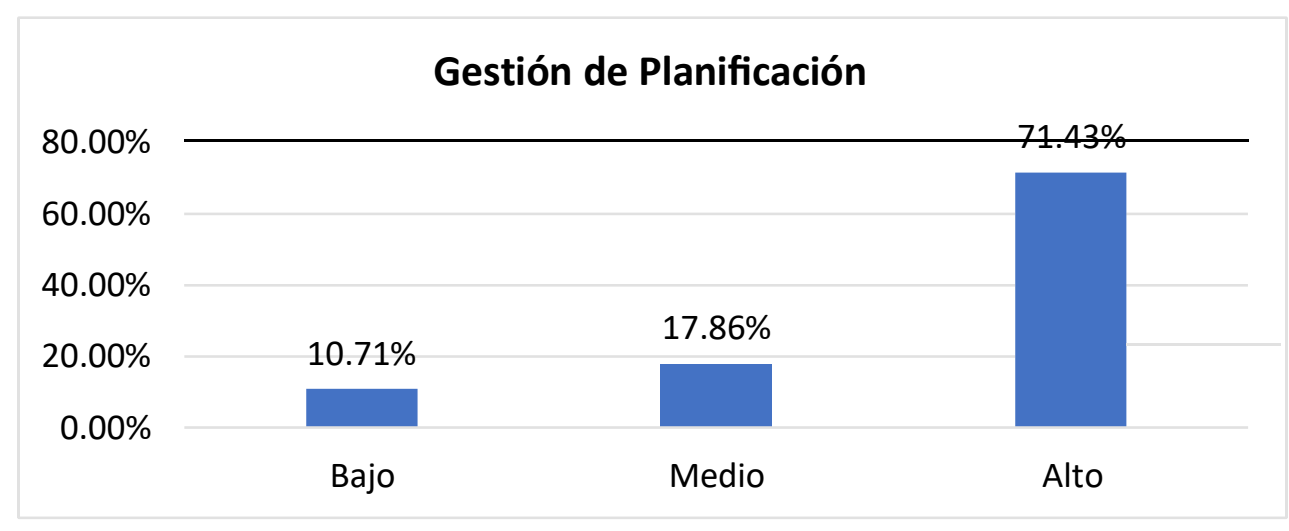

Se observó que el $71.43 \%$ de los docentes indicó niveles altos de gestión de planificación, seguido de un $17.86 \%$ que percibió niveles medio, y $10.71 \%$ indicaron niveles bajos. En general, el nivel de la dimensión gestión de planificación es bueno por encima del promedio.

\section{Tabla 8}

Nivel de la dimensión gestión de organización en los docentes de una institución educativa

\begin{tabular}{|c|c|c|c|c|c|}
\hline \multicolumn{6}{|c|}{ Gestión de Organización } \\
\hline & & rrecuencia & rorcentaje & $\begin{array}{l}\text { Porcentaje } \\
\text { válido }\end{array}$ & $\begin{array}{l}\text { Porcentaje } \\
\text { acumulado }\end{array}$ \\
\hline \multirow{4}{*}{ Válido } & Bajo & 4 & $14.29 \%$ & $14.29 \%$ & $14.29 \%$ \\
\hline & Medio & 7 & $25.00 \%$ & $25.00 \%$ & $39.29 \%$ \\
\hline & Alto & 17 & $60.71 \%$ & $60.71 \%$ & $100 \%$ \\
\hline & Total & 28 & $100 \%$ & $100 \%$ & \\
\hline
\end{tabular}

\section{Figura 7}

Nivel de la dimensión gestión de organización en los docentes de una institución educativa

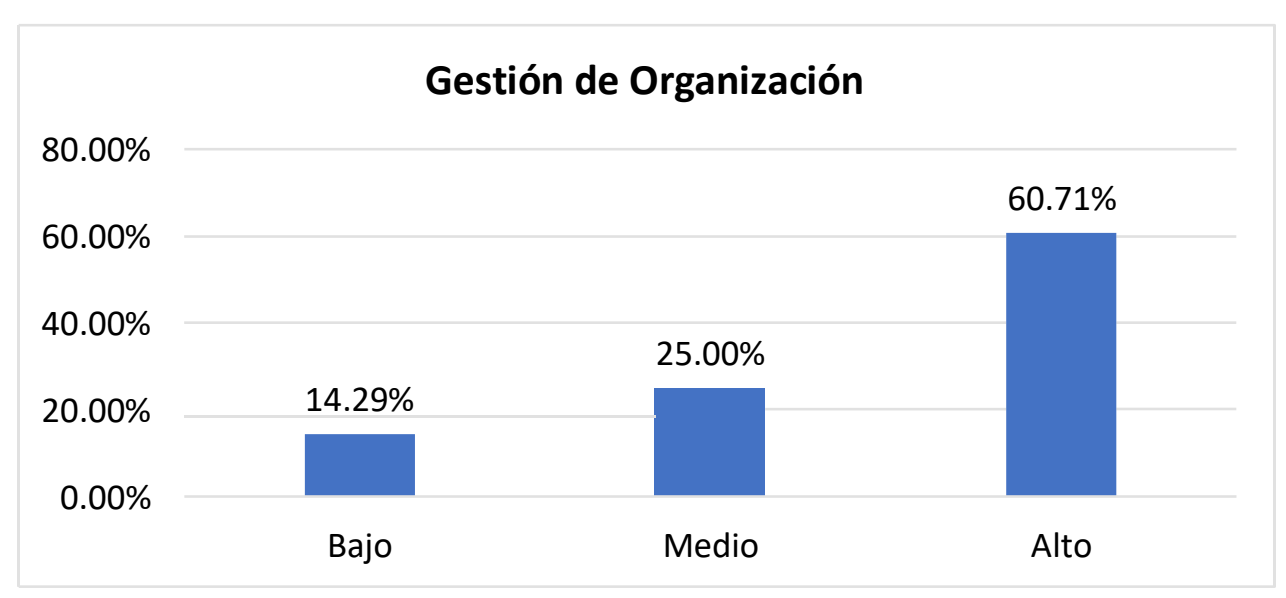

Según la tabla y figura anteriores, se observó que más de la mitad de docentes $(60.71 \%)$ señaló niveles altos de 
organización, el 25\% indicó niveles medios, y por último el $14.29 \%$ indicó niveles bajos. El nivel de organización es aceptable, pero puede mejorarse.

\section{Tabla 9}

Nivel de la dimensión gestión de dirección en los docentes de una institución educativa

\begin{tabular}{|c|c|c|c|c|c|}
\hline \multicolumn{6}{|c|}{ Gestión de Dirección } \\
\hline & & Frecuencix & rorcentaje & $\begin{array}{l}\text { Porcentaje } \\
\text { válido }\end{array}$ & $\begin{array}{l}\text { Porcentaje } \\
\text { acumulado }\end{array}$ \\
\hline \multirow{4}{*}{ Válido } & Bajo & 2 & $7.14 \%$ & $7.14 \%$ & $7.14 \%$ \\
\hline & Medio & 4 & $14.29 \%$ & $14.29 \%$ & $21.43 \%$ \\
\hline & Alto & 22 & $78.57 \%$ & $78.57 \%$ & $100 \%$ \\
\hline & Total & 28 & $100 \%$ & $100 \%$ & \\
\hline
\end{tabular}

\section{Figura 9}

Nivel de la dimensión gestión de dirección en los docentes de una institución educativa

\begin{tabular}{|c|c|c|c|}
\hline \multicolumn{4}{|c|}{ Gestión de Dirección } \\
\hline \multirow{3}{*}{$\begin{array}{l}80.00 \% \\
60.00 \%\end{array}$} & & & $78.57 \%$ \\
\hline & & & \\
\hline & & & \\
\hline $20.00 \%$ & $714 \%$ & $14.29 \%$ & \\
\hline $0.00 \%$ & 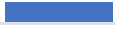 & & \\
\hline & Bajo & Medio & Alto \\
\hline
\end{tabular}

De la tabla y figura anteriores, se observó que un gran porcentaje de docentes $(78.57 \%)$ señaló niveles altos de dirección en la institución educativa, un 14.29\% señaló niveles medios y 7.14\% calificó como nivel bajo.

\section{Tabla 10}

Nivel de la dimensión gestión de control en los docentes de una institución educativa

\begin{tabular}{|c|c|c|c|c|c|}
\hline \multicolumn{6}{|c|}{ Gestión de Control } \\
\hline & & Frecuencic & Porcentaje & $\begin{array}{l}\text { Porcentaje } \\
\text { válido }\end{array}$ & $\begin{array}{l}\text { Porcentaje } \\
\text { acumulado }\end{array}$ \\
\hline \multirow{4}{*}{ Válido } & Bajo & 3 & $10.71 \%$ & $10.71 \%$ & $10.71 \%$ \\
\hline & Medio & 4 & $14.29 \%$ & $14.29 \%$ & $25.00 \%$ \\
\hline & Alto & 21 & $75.00 \%$ & $75.00 \%$ & $100 \%$ \\
\hline & Total & 28 & $100 \%$ & $100 \%$ & \\
\hline
\end{tabular}


Fernandez, D.

\section{Figura 10}

Nivel de la dimensión gestión de control en los docentes de una institución educativa

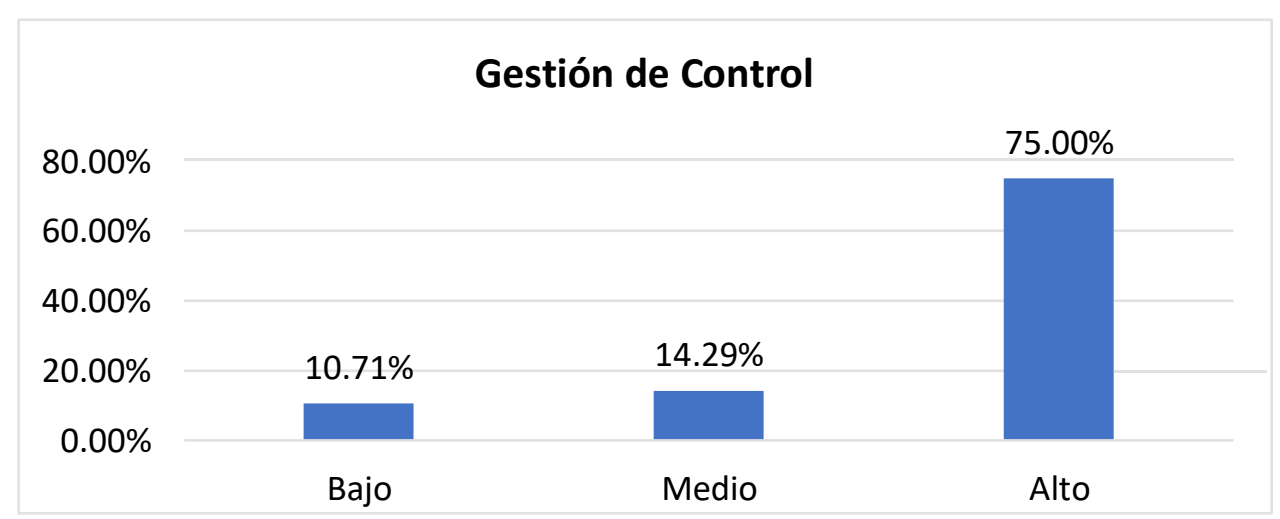

De la tabla y figura anteriores, se observó que el $75 \%$ de docentes calificaron que existen niveles altos de control en la institución educativa, el 14.29\% indicó niveles medios, en tanto el 10.71\% indicó niveles bajos.

\section{Desempeño Laboral}

\section{Tabla 11}

Nivel de desempeño laboral en los docentes de una institución educativa

\begin{tabular}{llllll}
\hline Desempeño laboral & & & & \\
\hline \multirow{6}{*}{ Válido } & rrecuencia & rorcentaje & $\begin{array}{l}\text { Porcentaje } \\
\text { válido }\end{array}$ & $\begin{array}{l}\text { Porcentaje } \\
\text { acumulado }\end{array}$ \\
\hline & Bajo & 3 & $10.71 \%$ & $10.71 \%$ & $10.71 \%$ \\
& Medio & 5 & $17.86 \%$ & $17.86 \%$ & $28.57 \%$ \\
& Alto & 20 & $71.43 \%$ & $71.43 \%$ & $100 \%$ \\
\hline
\end{tabular}

\section{Figura 11}

Nivel de desempeño laboral en los docentes de una institución educativa

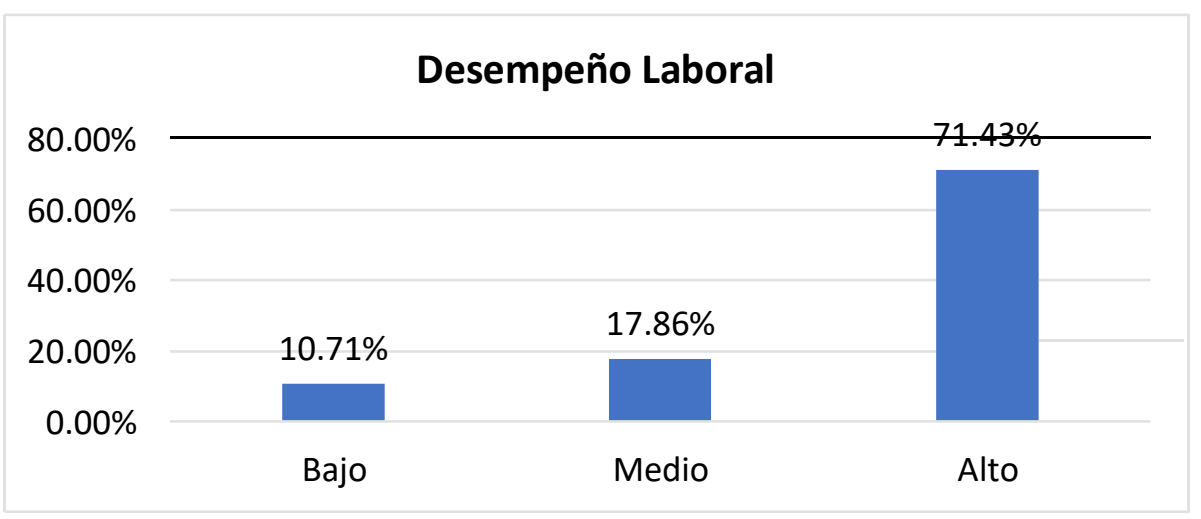


En el caso de esta variable y dimensiones, el coordinador calificó que el $71.43 \%$ de docentes presentan niveles altos de desempeño, el $17.86 \%$ niveles medios, y en tanto un $10.71 \%$ calificó como niveles bajos. En general el desempeño laborar es bueno, superior al promedio.

\section{Tabla 12}

Nivel de la dimensión desempeño de tareas en los docentes de una institución educativa

\begin{tabular}{llllll}
\hline Desempeño de tareas & & & & \\
\hline \multirow{4}{*}{ Válido } & rrecuenciz & rorcentaje & $\begin{array}{l}\text { Porcentaje } \\
\text { válido }\end{array}$ & $\begin{array}{l}\text { Porcentaje } \\
\text { acumulado }\end{array}$ \\
\hline & Bajo & 3 & $10.71 \%$ & $10.71 \%$ & $10.71 \%$ \\
& Medio & 4 & $14.29 \%$ & $14.29 \%$ & $25.00 \%$ \\
& Alto & 21 & $75.00 \%$ & $75.00 \%$ & $100 \%$ \\
& Total & 28 & $100 \%$ & $100 \%$ & \\
\hline
\end{tabular}

\section{Figura 12}

Nivel de la dimensión desempeño de tareas en los docentes de una institución educativa

\begin{tabular}{|c|c|c|c|}
\hline \multicolumn{4}{|c|}{ Desempeño de Tareas } \\
\hline $80.00 \%$ & & & $75.00 \%$ \\
\hline $60.00 \%$ & & & \\
\hline $40.00 \%$ & & & \\
\hline $20.00 \%$ & $10.71 \%$ & $14.29 \%$ & \\
\hline \multirow[t]{2}{*}{$0.00 \%$} & & & \\
\hline & Bajo & Medio & Alto \\
\hline
\end{tabular}

De la tabla y figuras anteriores, se calificó que $75 \%$ de los docentes en la institución educativa poseen niveles altos de desempeño de tareas, el $14.29 \%$ posee niveles medios de esta dimensión, seguido de un $10.71 \%$ que se calificó con niveles bajos.

\section{Tabla 13}

Nivel de la dimensión desempeño contextual en los docentes de una institución educativa

\begin{tabular}{|c|c|c|c|c|c|}
\hline \multicolumn{6}{|c|}{ Desempeño contextual } \\
\hline & & Frecuencia & Porcentaje & $\begin{array}{l}\text { Porcentaje } \\
\text { válido }\end{array}$ & $\begin{array}{l}\text { Porcentaje } \\
\text { acumulado }\end{array}$ \\
\hline \multirow{4}{*}{ Válido } & Bajo & 3 & $10.71 \%$ & $10.71 \%$ & $10.71 \%$ \\
\hline & Medio & 6 & $21.43 \%$ & $21.43 \%$ & $32.14 \%$ \\
\hline & Alto & 19 & $67.86 \%$ & $67.86 \%$ & $100 \%$ \\
\hline & Total & 28 & $100 \%$ & $100 \%$ & \\
\hline
\end{tabular}




\section{Figura 13}

Nivel de la dimensión desempeño contextual en los docentes de una institución educativa

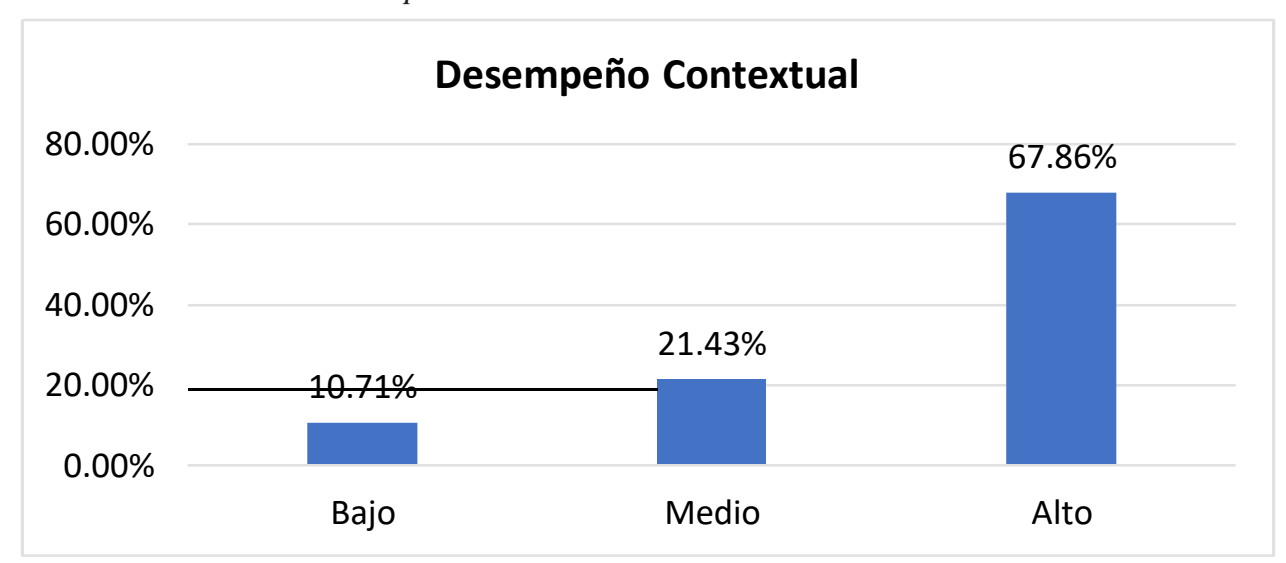

De la tabla y figura anteriores, el coordinador académico calificó que $67.86 \%$ de los docentes presentaron niveles altos de desempeño contextual, el $21.43 \%$ calificó con niveles medios, y por último un $10.71 \%$ presentó niveles bajos.

\section{Prueba de hipótesis}

\section{Hipótesis general}

$\mathrm{H}_{1}=$ Es probable que la gestión administrativa se relacione directa y significativamente con el desempeño laboral en una entidad educativa de Arequipa en la coyuntura de COVID-19.

$\mathrm{H}_{0}=$ Es probable que la gestión administrativa no se relacione directa y significativamente con el desempeño laboral en una entidad educativa de Arequipa en la coyuntura de COVID-19.

\section{Tabla 14}

Correlación entre gestión administrativa y desempeño laboral

\begin{tabular}{|c|c|c|c|}
\hline & & $\begin{array}{l}\text { Variable Gestión } \\
\text { Administratıva }\end{array}$ & $\begin{array}{l}\text { Variable } \\
\text { Desempeño } \\
\text { Laboral }\end{array}$ \\
\hline \multirow{2}{*}{$\begin{array}{l}\text { Variable } \\
\text { Aumınıstrauva }\end{array}$} & Gestión Correlación de Pearson & 1 &, 834 \\
\hline & Sig. (bilateral) & &, 000 \\
\hline \multirow{4}{*}{$\begin{array}{l}\text { Variable } \\
\text { Laboraı }\end{array}$} & $\mathrm{N}$ & 28 & 28 \\
\hline & Desempeño Correlación de Pearson & ,834 & 1 \\
\hline & Sig. (bilateral) & ,000 & \\
\hline & $\mathrm{N}$ & 28 & 28 \\
\hline
\end{tabular}

De acuerdo a nuestro coeficiente de correlación de Pearson, nos indica una correlación positiva alta. Por lo tanto, se rechaza la hipótesis nula y se acepta la hipótesis alterna. Es decir, la gestión administrativa se relaciona significativamente con el desempeño laboral en los docentes de una entidad educativa de Arequipa, 2020. 


\section{Hipótesis específicas}

$\mathrm{H}_{1}=$ Es probable que la Planificación de la Gestión Administrativa se relacione directa y significativamente con el Desempeño Laboral en una entidad educativa de Arequipa en la coyuntura de COVID-19.

\section{Tabla 15}

Correlación entre planificación de la gestión administrativa y desempeño laboral

\begin{tabular}{|c|c|c|c|}
\hline & & $\begin{array}{l}\text { Planificación de la } \\
\text { Gestión } \\
\text { Administrativa }\end{array}$ & $\begin{array}{l}\text { Variable } \\
\text { Desempeño } \\
\text { Laboral }\end{array}$ \\
\hline Planificación de la & Correlación de Pearson & 1 & ,795 \\
\hline Gestión Administrativa & $\begin{array}{l}\text { Sig. (bilateral) } \\
\mathrm{N}\end{array}$ & 28 & $\begin{array}{l}, 010 \\
28\end{array}$ \\
\hline Variable Desempeño & Correlación de Pearson & ,795 & 1 \\
\hline Laboral & $\begin{array}{l}\text { Sig. (bilateral) } \\
\mathrm{N}\end{array}$ & $\begin{array}{l}, 010 \\
28\end{array}$ & 28 \\
\hline
\end{tabular}

Se obtuvo como resultado un coeficiente de correlación de Pearson de 0.795 , esto indica una correlación positiva alta. Esto quiere decir que existe una relación significativa entre planificación de la gestión administrativa y desempeño laboral en docentes de una entidad educativa de Arequipa, 2020.

$\mathrm{H}_{2}=$ Es probable que la organización de la gestión administrativa se relacione directa y significativamente con el desempeño laboral en una entidad educativa de Arequipa en la coyuntura de COVID-19.

\section{Tabla 16}

Correlación entre organización de la gestión administrativa y desempeño laboral

\begin{tabular}{|c|c|c|c|}
\hline & & $\begin{array}{l}\text { Organización de la } \\
\text { Gestión } \\
\text { Administrativa }\end{array}$ & $\begin{array}{l}\text { Variable } \\
\text { Desempeño } \\
\text { Laboral }\end{array}$ \\
\hline Control de la Gestión & Correlación de Pearson & 1 & ,751 \\
\hline Administrativa & $\begin{array}{l}\text { Sig. (bilateral) } \\
\mathrm{N}\end{array}$ & 28 & $\begin{array}{l}, 005 \\
28\end{array}$ \\
\hline Desempeño & Correlación de Pearson & ,751 & 1 \\
\hline Laboral & $\begin{array}{l}\text { Sig. (bilateral) } \\
\text { N }\end{array}$ & $\begin{array}{l}, 005 \\
28\end{array}$ & 28 \\
\hline
\end{tabular}

Al examinarse los resultados obtenidos, el coeficiente de correlación de Pearson es igual a 0.751 , lo cual indica una correlación positiva alta. Esto quiere decir que la organización de la gestión administrativa se relaciona de manera significativa con el desempeño laboral en una entidad educativa de Arequipa, 2020. 
$\mathrm{H}_{3}=$ Es probable que la dirección de la gestión administrativa se relacione directa y significativamente con el desempeño laboral en una entidad educativa de Arequipa en la coyuntura de COVID-19.

\section{Tabla 17}

Correlación entre dirección de la gestión administrativa y desempeño laboral

\begin{tabular}{llll}
\hline & & $\begin{array}{l}\text { Dirección de la } \\
\text { Gestión } \\
\text { Administrativa }\end{array}$ & $\begin{array}{l}\text { Variable } \\
\text { Desempeño } \\
\text { Laboral }\end{array}$ \\
\hline $\begin{array}{l}\text { Dirección de la Gestión } \\
\text { Administrativa }\end{array}$ & $\begin{array}{l}\text { Correlación de Pearson } \\
\text { Sig. (bilateral) }\end{array}$ & 1 &, 736 \\
Variable & $\mathrm{N}$ & 28 &, 002 \\
Laboral & Desempeño & Correlación de Pearson &, 736 \\
& Sig. (bilateral) &, 002 & 1 \\
& $\mathrm{~N}$ & 28 & 28 \\
\hline
\end{tabular}

De acuerdo a los resultados obtenidos, tenemos un coeficiente de correlación de Pearson de 0.736 , indicando una correlación positiva alta. Lo cual quiere decir que existe una relación significativa entre dirección de la gestión administrativa y el desempeño laboral en una entidad educativa de Arequipa, 2020.

$\mathrm{H}_{4}=$ Es probable que el control de la gestión administrativa se relacione directa y significativamente con el desempeño laboral en una entidad educativa de Arequipa en la coyuntura de COVID-19.

\section{Tabla18}

Correlación entre control de la gestión administrativa y desempeño laboral

\begin{tabular}{|c|c|c|c|}
\hline & & $\begin{array}{l}\text { Control de la Gestión } \\
\text { Aamınıstratıva }\end{array}$ & $\begin{array}{r}\text { Variable } \\
\text { Laboral }\end{array}$ \\
\hline \multirow{3}{*}{$\begin{array}{l}\text { Control de la Gestión } \\
\text { Aamınıstratıva }\end{array}$} & Correlación de Pearson & 1 &, 760 \\
\hline & Sig. (bilateral) & & ,000 \\
\hline & $\mathrm{N}$ & 28 & 28 \\
\hline \multirow{3}{*}{$\begin{array}{l}\text { Variable Desempeño } \\
\text { Laboraı }\end{array}$} & Correlación de Pearson &, 760 & 1 \\
\hline & Sig. (bilateral) & 000 & \\
\hline & $\mathrm{N}$ & 28 & 28 \\
\hline
\end{tabular}

$\mathrm{Al}$ examinar los resultados obtenidos, tenemos un coeficiente de correlación de Pearson $=0.760$, lo cual indica una correlación positiva alta. Esto nos quiere decir que el control de la gestión administrativa se relaciona significativamente con el desempeño laboral en una entidad educativa de Arequipa, 2020.

\section{DISCUSIÓN}

La investigación tuvo como fin determinar la relación entre las variables de gestión administrativa y el desempeño laboral en el personal docente de la entidad educativa ubicada en la ciudad de Arequipa, la misma que se llevó a cabo con la colaboración de 28 docentes. Luego del análisis y las pruebas estadísticas aplicadas a la muestra, se comprueba que la gestión administrativa influye positiva y significativamente en el desempeño de los docentes de la Institución Educativa "Andenes de Chilina"; esto porque, según los resultados estadísticos obtenidos, se obtuvo una correlación significativa de r= 0.834 para la hipótesis general, representando así una alta relación significativa entre las variables. 
De igual manera, respecto a las hipótesis específicas, existe una relación positiva y significativa entre cada una de las dimensiones de gestión administrativa versus la variable desempeño laboral, ubicándose el coeficiente de correlación de Pearson en un rango de 0.736 y 0.795 . En general los niveles de ambas variables son aceptables, debido a que hay buenas condiciones laborales en la entidad educativa. Cabe mencionar que debido a esta coyuntura COVID-19, los docentes fueron capacitados en el uso de las TICs, especialmente la plataforma de Google Meet para brindar una enseñanza de calidad. También existe una predisposición del director de la institución educativa a escuchar opiniones. Un punto que destacar es que en la entidad existen algunas falencias respecto a la organización, se presentan algunas descoordinaciones y en ocasiones algunas de las funciones no están claras. Para corroborar los resultados obtenidos, es necesario hacer una comparación con otros estudios, como se detalla a continuación.

Monroy (2013) llegó a la conclusión que en la institución en donde llevo a cabo su estudio, debido al tipo de liderazgo ejercido (autoritario), los maestros se desempeñaban por presión; lo cual influyó de manera negativa en su desempeño. Por otro lado, se demuestra también como la gestión educativa influye de manera positiva en el desempeño docente del nivel secundario de la I.E. Modelo de Trujillo.

Por su parte, Meza Revatta (2019), en su investigación confirmó una relación positiva moderada entre gestión administrativa y desempeño docente en las instituciones Colegio Adventista del Titicaca, Colegio Adventista Americana y Colegio Adventista Belén de la ciudad de Juliaca. Demostró que a mayor eficacia de la gestión administrativa: buen manejo de recursos económicos, cumplimiento de la normatividad y de las funciones, hay una mejor tendencia a adquirir un mejor desempeño docente.

Quiroz \& Vega (2020) llevaron a cabo su investigación en la Institución Educativa Nº 80826 "Miguel Grau", Huaranchal, donde se obtuvo un coeficiente Rho de Spearman= 0,567, evidenciando que existe relación directa significativamente moderada entre la gestión administrativa y el desempeño docente, destacando que resulta importante tratar de identificar los otros factores que estarían relacionados con el desempeño docente en la institución educativa que fue objeto de análisis.

Yábar (2013) desarrolló su investigación en la Institución Educativa Privada Santa Isabel de Hungría de la Ciudad de Lima-Cercado. Como resultado, al concluir su estudio, obtuvo que existe una relación directa entre la gestión educativa y la práctica docente. Los resultados señalan que es necesario que los directivos y los docentes coordinen actividades conjuntas que les permitan tener una participación conjunta en la toma de decisiones para el cumplimiento de los objetivos de la institución.

Finalmente, Rosario (2017) realizó la investigación para determinar: "si la gestión educativa se relaciona con el desempeño docente de educación secundaria de la Institución Educativa Augusto B. Leguía, distrito Puente Piedra", Lima, en el año 2016. La hipótesis se verificó con las tablas de Rho Spearman donde los resultados obtenidos demuestran que existe relación significativa entre la gestión educativa y el desempeño docente, logrando un nivel de $r=0,837$.

Las investigaciones anteriormente mencionadas han logrado demostrar los objetivos de estudio, lo cual será de utilidad para posteriores estudios y de igual manera poder establecer conclusiones.

\section{CONCLUSIONES}

Se demostró que existe una relación significativa entre las variables gestión administrativa y desempeño laboral de los docentes de la Institución Educativa "Andenes de Chilina", Arequipa. Siendo que se obtuvo un coeficiente de correlación de Pearson de 0.834 , con nivel de significancia $\mathrm{p}=0.000$, siendo esto menor al $5 \%(\mathrm{p}<0.05)$ representando una alta asociación entre las variables.

Se determinaron los niveles de las variables gestión administrativa y desempeño laboral de los docentes de la Institución Educativa “Andenes de Chilina”, Arequipa, encontrándose ambas en niveles aceptables.

Se evidenció que la planificación de la gestión administrativa se relaciona significativamente con el desempeño laboral docente en la Institución Educativa "Andenes de Chilina", Arequipa. El coeficiente de correlación de Pearson es 0.795 con un nivel de significancia de 0.010 , siendo esto menor al $5 \%(\mathrm{p}<0.05)$. 
Se demostró que la organización de la gestión administrativa se relaciona significativamente con el desempeño laboral docente en la Institución Educativa "Andenes de Chilina", Arequipa. El coeficiente de correlación de Pearson es 0.751 con un nivel de significancia de 0.005 , siendo esto menor al $5 \%$ ( $\mathrm{p}<0.05)$.

Igualmente, se demostró que la dirección de la gestión administrativa se relaciona significativamente con el desempeño laboral docente en la Institución Educativa "Andenes de Chilina", Arequipa. El coeficiente de correlación de Pearson es 0.736 con un nivel de significancia de 0.002 , siendo esto menor al $5 \%$ ( $p<0.05$ ).

Asimismo, se demostró que el control de la gestión administrativa se relaciona significativamente con el desempeño laboral docente en la Institución Educativa "Andenes de Chilina", Arequipa. El coeficiente de correlación de Pearson es 0.760 con un nivel de significancia de 0.000 , siendo esto menor al $5 \%$ ( $<<0.05$ ).

Después de analizar los resultados obtenidos, si bien son positivos en todos los ámbitos, es necesario prestar especial atención a la organización en la entidad educativa. A pesar de que obtuvieron resultados mayores al promedio, se presentan algunas falencias que es necesario mejorarlas, como las descoordinaciones que se vienen dando meses previos a la coyuntura COVID-19.

\section{REFERENCIAS}

Benites, L. y Castillo, D. (2018). Percepción de la gestión administrativa y el desempeño laboral del docente de una I.E.P de nivel secundario de la Rinconada Trujillo 2017. [Tesis de Maestría, Universidad César Vallejo, Trujillo].

Carbajal, M. (2019). Clima organizacional y satisfacción laboral en la Institución Educativa Parroquial Santa Rita del distrito de Cotabambas - Apurímac. [Tesis de Maestría, Universidad Nacional de San Agustín, Arequipa].

Chávez, M. \& Olivos, M. (2019). Gestión Educativa y Desempeño Docente, en la institución educativa “Modelo" Trujillo, 2018. [Tesis de Maestría, Universidad Católica de Trujillo, Trujillo].

Hernández, R., Fernández, C., \& Baptista, L. (2014). Metodología de la investigación (Sexta ed.). McGraw-Hill.

Meza Revatta, L. (2019). Gestión educativa y desempeño docente en instituciones educativas adventistas de la ciudad de Juliaca, 2018. [Tesis de Maestría, Universidad Peruana Unión, Lima].

Monroy, J. (2013). Liderazgo directivo como elemento estratégico en la efectividad del desempeño docente. [Tesis de Maestría, Universidad de Carabobo, Carabobo].

Quiroz, L. \& Vega, Y. (2020). Gestión administrativa y desempeño docente en la Institución educativa pública de La Libertad, 2019. [Tesis de Maestría, Universidad Católica de Trujillo Benedicto XVI, Trujillo].

Rodríguez, A. \& Pérez, A. (2017). Métodos científicos de indagación y de construcción del conocimiento. Revista Escuela Administración de Negocio (82), 1-26. https://www.redalyc.org/pdf/206/20652069006.pdf

Rosario, A. (2017). La gestión educativa y el desempeño docente de educación secundaria de la institución educativa Augusto B. Leguía, distrito Puente Piedra-Lima-2016. [Tesis de Maestría, Universidad Nacional Mayor de San Marcos, Lima].

Tacuche Mariño, B. (2018). Gestión administrativa y calidad de servicio de la Institución Educativa $N^{\circ}$ 32282 “San Miguel”, Lauricocha, 2018. [Tesis de Maestría, Universidad César Vallejo, Huánuco].

Vaillant, D. (2009). Mejorando la formación y el desarrollo profesional docente en Latinoamérica. Revista Pensamiento Educativo, II(41), 207-222.

Yábar, I. (2013). La Gestión educativa y su relación con la práctica docente en la Institución Educativa Privada Santa Isabel de Hungría de la Ciudad de Lima-Cercado. [Tesis de Maestría, Universidad Nacional Mayor de San Marcos, Lima]. 